\title{
Effect of mobile telephone reminders on treatment outcome in HIV: evidence from a randomised controlled trial in India
}

\author{
(c) $(1)$ (8)
}

\author{
Anita Shet associate professor ${ }^{12}$, Ayesha De Costa associate professor ${ }^{2}$, N Kumarasamy chief \\ medical officer $^{3}$, Rashmi Rodrigues assistant professor ${ }^{24}$, Bharat Bhusan Rewari senior consultant ${ }^{5}$, \\ Per Ashorn professor ${ }^{6}$, Bo Eriksson professor ${ }^{2}$, Vinod Diwan professor ${ }^{2}$, and the HIVIND Study \\ Team
}

${ }^{1}$ Department of Pediatrics, St John's Medical College Hospital, Bangalore 560034, India; ${ }^{2}$ Department of Public Health Sciences, Karolinska Institute, Stockholm 17177, Sweden; ${ }^{3}$ YRG Centre for AIDS Research and Education, Chennai 600113, India; ${ }^{4}$ Department of Community Medicine, St John's Medical College Hospital, Bangalore 560034, India; ${ }^{5}$ National AIDS Control Organization, Department of AIDS Control, Ministry of Health and Family Welfare, Government of India, New Delhi 110001 , India; ${ }^{6}$ Department for International Health, University of Tampere School of Medicine, Tampere, Finland

\begin{abstract}
Objective To assess whether customised mobile phone reminders would improve adherence to therapy and thus decrease virological failure among HIV infected patients starting antiretroviral treatment (ART).

Design Randomised controlled trial among HIV infected patients initiating antiretroviral treatment.
\end{abstract}

Setting Three diverse healthcare delivery settings in south India: two ambulatory clinics within the Indian national programme and one private HIV healthcare clinic.

Participants 631 HIV infected, ART naïve, adult patients eligible to initiate first line ART were randomly assigned to mobile phone intervention $(n=315)$ or standard care $(n=316)$ and followed for 96 weeks. Intervention The intervention consisted of customised, interactive, automated voice reminders, and a pictorial message that were sent weekly to the patients' mobile phones for the duration of the study.

Main outcome measures The primary outcome was time to virological failure (viral load $>400$ copies $/ \mathrm{mL}$ on two consecutive measurements). Secondary outcomes included ART adherence measured by pill count, death rate, and attrition rate. Suboptimal adherence was defined as mean adherence $<95 \%$.

Results Using an intention-to-treat approach we found no observed difference in time to virological failure between the allocation groups: failures in the intervention and standard care arms were 49/315 (15.6\%) and $49 / 316(15.5 \%)$ respectively (unadjusted hazard ratio $0.98,95 \%$ confidence interval 0.67 to $1.47, \mathrm{P}=0.95$ ). The rate of virological failure in the intervention and standard care groups were 10.52 and 10.73 per 100 person years respectively. Comparison of suboptimal adherence was similar between both groups (unadjusted incidence rate ratio 1.24 $95 \% \mathrm{Cl} 0.93$ to $1.65, \mathrm{P}=0.14$ ). Incidence proportion of patients with suboptimal adherence was $81 / 300(27.0 \%)$ in the intervention arm and $65 / 299(21.7 \%)$ in the standard care arm. The results of analyses adjusted for potential confounders were similar, indicating no significant difference between the allocation groups. Other secondary outcomes such as death and attrition rates, and subgroup analysis also showed comparable results across allocation groups.

Conclusions In this multicentre randomised controlled trial among ART naïve patients initiating first line ART within the Indian national programme, we found no significant effect of the mobile phone intervention on either time to virological failure or ART adherence at the end of two years of therapy.

Trial registration Current Controlled Trials ISRCTN79261738.

\section{Introduction}

The current explosion in the use of mobile phone technology in healthcare coupled with decreasing costs of wireless communications worldwide has led to a panoply of promising mobile phone based interventions among patients with chronic conditions, including HIV infection. ${ }^{1}$ Systematic reviews acknowledge that mobile phone interventions can help improve specific health conditions, but they also underline the need for high quality clinical trials measuring outcomes in real world 
settings. ${ }^{23}$ Recent positive reports from Kenya ${ }^{45}$ led a Cochrane review panel to conclude that mobile phone based reminder systems can be beneficial in HIV treatment. ${ }^{6}$ Recognising the promise of mobile phones in public health, as suggested by these early reports, the World Health Organization in its 2013 guidelines encourages national programmes to explore the scale up of mobile phone interventions. ${ }^{7}$ This approach, however, merits caution and a deeper examination of available evidence, particularly as conflicting trial results have been reported from other settings such as Cameroon and Brazil. ${ }^{8}{ }^{9}$ The absence of a clear evidence base for scale up can limit the potential of this tool to translate into effective health outcomes. ${ }^{10}$

With an estimated 2.5 million Indian people living with HIV infection, and an expanding ambulatory population receiving antiretroviral treatment (ART), there is a need in India to sustain treatment success. ${ }^{11}$ The appeal of mobile phones to enhance adherence to treatment was clear, but their implementation was limited by a lack of strong evidence to support their use in an Indian setting, as well as by inconsistent reports from other settings. We hypothesised that customised motivational low cost mobile phone reminders would enhance adherence to ART among Indian patients. This hypothesis, grounded in the social cognitive theory of planned behaviour ${ }^{12}$ was based on an initial feasibility study on patients' perceptions of mobile phone reminders ${ }^{13}$ and a subsequent pilot study that demonstrated a beneficial effect on adherence up to six months after the mobile phone intervention was discontinued. ${ }^{14}$ We then tested our hypothesis using a rigorous randomised controlled trial design among HIV infected patients initiating ART in southern India. ${ }^{15}$

\section{Methods}

\section{Trial design}

This was a multicentre randomised controlled open label trial conducted in south India. The rationale, study design, and conduct of statistical analysis of the HIV-India (HIVIND) study have been published previously, ${ }^{15}$ and a summary of protocol details are available at http://ec.europa.eu/research/health/ infectious-diseases/poverty-diseases/projects/190_en.htm. ${ }^{16}$ Patients and the randomisation team were aware of the intervention assignment; while research staff assessing patients, laboratory staff, statisticians, and authors were blind to the allocation. Allocations were revealed only after the blinded results were analysed and discussed by all authors.

\section{Ethical considerations}

Ethical approvals for the conduct of the trial were obtained from all participating institutions prior to study initiation (St John's Medical College Hospital, Bangalore (IERB 1/369/08-92/2008); Mysore Medical College and Research Institute

(NO/PS/173/2010); YRGCARE Medical Center, Chennai (IRB April18/2009); Karolinska Institutet, Stockholm (Protokoll 2009/303-31/2); University of Tampere (R0906)). In accordance with national requirements and the principles of the Declaration of Helsinki, written informed consent was obtained from all participants prior to enrollment. Confidentiality was maintained at all levels of data management. An independent data safety and monitoring board reviewed the data and performed a pre-specified blinded interim analysis midway through the trial.

\section{Study setting and participants}

Patients with documented HIV infection attending the ambulatory clinics from three sites in two Indian states with a high prevalence of HIV infection were recruited. The two sites in Karnataka State were part of the National AIDS Control Program, and included St John's Medical College Hospital, Bangalore, a missionary teaching hospital with an ART centre that is run as a public-private partnership, and Krishna Rajendra Hospital, Mysore, a government run tertiary care hospital providing free healthcare. The third site, YRGCARE Medical Centre, Chennai, is a private non-governmental centre in Tamil Nadu State. Together these sites catered to both urban and rural populations in south India, providing multidisciplinary care, counselling, and treatment for over $10000 \mathrm{HIV}$ infected patients at the time of study initiation.

Eligible patients included HIV infected individuals with adequate documentation of their HIV positive status, aged 18-60 years, ART naïve, and meeting the criteria for start of first line ART as per the 2007 Indian national guidelines. ${ }^{17}$ We excluded those patients who tested positive for HIV-2, were severely ill (Karnofsky score $<70$ ), expressed inability to attend all study visits, had no mobile phone network in their area of residence, or had another member of their household already recruited into the same study (to minimise intervention "contamination").

\section{Randomisation}

Participants were randomly assigned in a 1:1 allocation ratio to the mobile phone intervention arm or control arm.

Randomisation was performed stratified for sex, in permuted blocks of four or six. Sequentially numbered opaque sealed envelopes were used as a method of allocation concealment. All patients were given a mobile phone in order to obviate any potential effect the possession of the phone might have on adherence; but only those in the intervention arm received phone reminders.

\section{The mobile phone intervention}

The main aspect of the intervention was a customised motivational voice call that went out once a week at a time selected by each patient. The patient also chose the sex and language of the pre-recorded voice call. This automated call began with a greeting and the hope that the patient was feeling well, followed by an inquiry whether medications were taken as prescribed. The message was considered interactive or bidirectional, since it required the patient to respond to a question about the previous day's pill doses, by pressing "1" for yes or "2" for no. If the patient failed to respond to the call, a maximum of three more calls were made over the ensuing 24 hours until a response was obtained. The second aspect of the intervention included a weekly non-interactive neutral pictorial message sent out as a reminder four days after the automated call.

\section{Standard care}

All participants in the control and intervention arms received standard care, based on national guidelines. ${ }^{17}$ This included up to three counseling sessions prior to initiation of ART, routine clinical and laboratory tests at baseline, and follow-up assessments every six months. First line ART regimens included those based on zidovudine, stavudine, or tenofovir, along with lamivudine and either nevirapine or efavirenz, and were dispensed free of cost as generic fixed-dose combination pills every 1-3 months.

\section{Study procedures}

Participants returned for study visits at weeks 2, 8, and 12 after ART initiation, and subsequently every 12 weeks until week 96 or until the point of virological failure. All study visits were 
integrated with routine clinic visits as per the national guidelines. At every study visit, medical details were documented. A researcher not involved in routine care and who was blinded to the allocation, measured pill count adherence. Laboratory assessments included CD4 cell count and viral load every three months, and other tests as clinically indicated. A quality control manager ensured that data entered in the case report forms were complete and accurate. A double data entry system was employed to minimise errors, which was supervised by a dedicated data manager. The fidelity of the intervention was monitored continuously throughout the trial by checking the mobile phone intervention software reports that were available on a daily basis. In addition, selected research staff also received the intervention and maintained a weekly report for quality control.

\section{Study outcomes and definitions}

The primary outcome in the trial was time to virological failure (defined as plasma viral load $>400$ copies $/ \mathrm{mL}$ on two consecutive samples measured at least one month apart) six months after initiating ART. The secondary outcome was patient adherence to ART using pill count expressed as a percentage of pills taken, divided by total pills dispensed. This measurement also accounted for returned or lost pills. Adherence was measured during all scheduled study visits starting from week 4 after ART initiation until end of study or until endpoint was reached (weeks 4,8 , and 12, and then every 12 weeks until week 96). Optimal adherence was defined as mean adherence $\geq 95 \%$ during the follow-up period. Attrition and death rates were included as secondary outcomes.

\section{Sample size calculation}

To estimate the necessary numbers of patients in each of the two allocation groups, we assumed the risk for virological failure in the standard care arm to be $10 \%$ (risk rate 0.1 ) during the two years, while the intervention was expected to reduce this risk to $3 \%(0.03) .{ }^{18}$ A total sample of 532 patients (266 in each arm) would provide $90 \%$ power to detect such a risk difference in a two-sided log-rank test with significance level of 0.05. Expecting an attrition rate of $10 \%$, the trial was planned to have a minimum of 600 patients.

\section{Statistical analysis}

Trial analysis was performed using an intention-to-treat principle that included all originally randomised patients. Additional per protocol analysis was performed excluding patients with major protocol deviations (number of patients who were lost to follow-up or withdrew consent; 24 and 30 in the intervention and standard care arms respectively). Time-to-event analyses - which included Kaplan-Meier survival curves, log rank test, and Cox proportional hazards models-were used to compare intervention and standard care groups with respect to virological failure. Cox regression analysis was performed adjusting for site and other sociodemographic confounders.

The secondary outcome of mean pill count adherence was dichotomised to a binary variable with outcomes $<95 \%$ and $\geq 95 \%$. Crude and adjusted incidence rate ratios (incidence rate of patients with mean adherence $\geq 95 \%$ ) between the two arms were calculated using Poisson regression model.

Stratified analyses with regard to primary and secondary outcomes were performed on predefined subgroups based on site, age, presence of transmitted drug resistance, ART regimen, and level of adherence. The consistency of the intervention effect among these subgroups was assessed by formal tests of interaction. STATA v13 software was used for all analyses. A $P$ value of $<0.05$ was considered to denote statistical significance, and all tests were two sided.

\section{Results \\ Patients}

Between July 2010 and August 2011, 1140 HIV infected, ART naïve individuals were screened, and 509 ineligible participants excluded (fig $1 \Downarrow$ ). Among the 631 enrolled participants, 273 $(43.3 \%)$ were women, $286(45.3 \%)$ resided in a rural area, 515 $(81.6 \%)$ had high school level education and beyond, and 495 (78.45\%) owned or shared mobile phones. After enrolment, 315 and 316 participants were randomised to the intervention and standard care arms respectively. Both groups had similar pre-randomisation characteristics (table $1 \Downarrow$ ).

\section{Study outcomes}

\section{Primary outcome}

The rate of virological failure in the intervention and standard care groups were 10.52 (95\% confidence interval 8.11 to 14.19 ) and 10.73 (7.95 to 13.92$)$ per 100 person years respectively. The number of virological failures in the intervention and standard care arms were 49/315 (15.6\%) and 49/316 (15.5\%) respectively (table $2 \Downarrow$ ). There was no observed statistically significant difference in the time to virological failure between the allocation groups (unadjusted hazard ratio $0.98,95 \% \mathrm{CI}$ 0.67 to $1.47, \mathrm{P}=0.95$ ) (fig $2 \Downarrow$ ). Adjustment in a Cox proportional hazards model for covariates such as site, age, sex, rural residence, education level, previous experience with mobile phones, baseline CD4 count, ART regimen, occurrence of adverse drug reactions, and presence of transmitted drug resistance at baseline did not reveal any significant difference between the intervention and standard care arms (adjusted hazard ratio $0.96,0.65$ to $1.43, \mathrm{P}=0.85$ ) (table $2 \Downarrow$ ). A similar lack of significant difference was seen in the per protocol analysis (adjusted HR 0.96, 0.64 to $1.43, \mathrm{P}=0.89$ ). Failure rates across allocation groups remained similar at 6,12 , and 18 months after ART initiation.

\section{Secondary outcomes}

The incidence proportion of patients with suboptimal adherence was $81 / 300(27.0 \%)$ in the intervention arm and 65/299 (21.7\%) in the standard care arm (table 2). Suboptimal adherence rates did not differ significantly between the intervention and standard care groups (crude incidence rate ratio $1.24,95 \%$ CI 0.93 to $1.65, \mathrm{P}=0.14)$. When adjusted for covariates in a Poisson model, no change was seen (adjusted incidence rate ratio 1.24, 0.94 to $1.63, \mathrm{P}=0.13$ ). Comparable results were obtained in the per protocol analysis (adjusted incidence rate ratio 1.26, 0.94 to $1.69, \mathrm{P}=0.13$ ). Both allocation groups had similar proportions of patients with suboptimal adherence over the course of the trial at 6,12 , and 18 months.

Deaths numbered 21/315 (6.7\%) and 23/316 (7.3\%) in the intervention and standard care groups respectively. Mortality assessment showed that 4.51 (95\% CI 2.94 to 6.91) deaths per 100 person years occurred in the intervention arm and 5.04 (3.35 to 7.58 ) deaths per 100 person years occurred in the standard care arm. In each arm, 24/315 (7.6\%) and 30/316 (9.5\%) were lost to follow up. Attrition occurred at the rate of 3.43 (95\% CI 2.10 to 5.61 ) and 4.82 (3.17 to 7.31 ) per 100 person years in the intervention and standard care arms respectively.

Comparison of death rates (adjusted incidence rate ratio 0.91, 0.51 to $1.60, \mathrm{P}=0.74$ ) and attrition rate (adjusted incidence rate 
ratio $0.59,0.32$ to $1.10, \mathrm{P}=0.10)$ did not differ significantly between allocation groups (table $2 \Downarrow$ ).

\section{Subgroup analysis}

Significant differences in virological failure or adherence rates between allocation groups were consistently absent across subgroups of site, age, level of baseline immunosuppression, ART regimen, presence of transmitted drug resistance, and level of mean adherence to therapy (fig $3 \Downarrow$ ). Tests of interaction did not reveal any difference across subgroups. There were site differences in mean adherence levels (proportion optimally adherent in Bangalore 89\%, in Mysore 67\%, in Chennai 73\%, $\mathrm{P}<0.001$ ), but the rate of virological failure was similar across the intervention and standard care arms at each site.

\section{Fidelity of the intervention}

A high level of intervention fidelity was maintained during the trial. The mobile phone monitoring reports of the total number of calls that went out to all patients in the intervention arm indicated that $97 \%$ of expected calls went through, and $86 \%$ of these calls were received by patients (supplementary fig 3 ). In addition, at an individual level, the patients in the intervention arm who did not receive the phone calls were monitored, and they constituted a similar percentage when compared with the analysis of total calls. These patients were administered questionnaires about the reasons for non-receipt of calls; chief reasons for non-receipt of calls were technical (poor network or handset problems, 24\%) and personal reasons (being busy or away from the phone, $55 \%$; phone being switched off, $18 \%$; or changed numbers, $3 \%$ ). There were no reported adverse events associated with phone use, although concerns about intrusiveness and loss of privacy were expressed by six of 286 patients in the intervention arm who responded to a questionnaire about the phone intervention at the end of their follow-up period.

\section{Discussion}

In this multicentre randomised controlled trial among ART naïve patients initiating first line ART within the Indian national programme, we were unable to detect a significant effect of mobile phone voice reminders on either time to virological failure or adherence to treatment at the end of two years of therapy. We also found no significant effect within relevant subgroups and at different time points during the trial.

\section{Strengths and limitations of study}

Our trial sought to ensure a high degree of internal validity by random assignment, use of robust outcome measures, blinded assessment of adherence and laboratory values, and pre-specified blinded analyses. The inclusion of diverse care delivery settings and nesting of the trial within the Indian national AIDS control programme, simulated a "real world" setting, and contributed to external validity. Further, clinical and sociodemographic features of our study population matched characteristics of the general HIV infected population of India treated with ART. ${ }^{11} 1920$ Virological failure rate seen in this study was consistent with reported rates in other resource limited settings among patients receiving similar first line ART. ${ }^{21-23}$ ART adherence was rigorously measured using pill count, an objective method that has economic and clinical advantages in resource limited settings. This method is not subject to potential biases related to patient self reporting such as desirability and recall bias, ${ }^{24}$ although inaccuracies can occur from pill dumping or unreported pill losses.
Trial limitations included a low representation of patients with other modes of HIV transmission such as injectable drug use, since the predominant mode of HIV acquisition in southern India is via heterosexual transmission. The lack of complete blinding can also be considered as a limitation, as the trial design and nature of the intervention necessitated that the patients could not be blinded to the allocation. The overall high mean adherence may have created a ceiling effect, limiting the scope of the intervention to show a positive effect. Adherence studies from India indicated that the proportion of patients with $\geq 95 \%$ adherence ranged from $65 \%$ to $97 \% .{ }^{25}{ }^{26}$ The high adherence level seen in our population might have also been partly due to a Hawthorne effect (improved clinical outcome in the control and intervention arms by virtue of trial participation and increased surveillance), which would have diminished the ability to detect a true difference in the trial. ${ }^{27} \mathrm{~A}$ stratified analysis among patients with low adherence (mean adherence $<90 \%$ and $<80 \%$ ) was consistent with the overall result of no intervention effect, although this will not adequately account for the Hawthorne effect, if present.

Although a randomised controlled trial design is generally a robust design that controls for unknown confounders and can often provide a causal link, there are inherent biases that may be particularly relevant in behavioural intervention trials. When both the intervention (in this case, mobile phone response) and outcome (adherence to medication) have a strong behavioural component, the act of research participation itself may account for the observed change more than the intended intervention being studied. ${ }^{28}$ Possible factors that could have contributed to behaviour change in both intervention and control groups include signing a consent form, being administered study questionnaires, rigorous adherence measurements at every study visit, receiving a mobile phone plus wage compensation for participating in the trial, and being subjected to extra blood tests for CD4 cell counts and viral load testing.

Power analysis for the trial was based on the estimated risk for medication failure in the standard care arm. The observed failure rate was similar to the estimated rate. It is likely that the power calculation overestimated the effect size, assuming that a $70 \%$ reduction is likely to occur in the intervention arm. However the number of failures were exactly the same in both arms, and hence the possibility of a type II error was minimal. The phenomenon of non-consenters may also be considered a source of bias in all trial settings; among the 139 (18\%) patients who declined consent, demographic and clinical features were similar to those of the patients who participated in the trial, indicating that external validity of the results is likely to be in the acceptable range for these known factors. It is conceivable that participation in the trial, and receipt of regular voice and text messages, could have contributed to a risk of stigma for the participants; however, exploring this will require more in-depth qualitative studies.

\section{Comparison with other studies}

Two recent studies have provided much momentum for the spreading pre-eminence of mobile phones in HIV healthcare. ${ }^{45}$ A careful examination of these results revealed new insights into the analysis methods and the possibility of reporting bias. The first Kenyan trial ${ }^{4}$ assessed the effect of interactive weekly text message contact with patients, and reported, in an intention-to-treat analysis, a significant positive effect on a subjective measure of self reported adherence at one year. However the effect on virological failure was uncertain, with wide confidence intervals (adjusted odds ratio $0.71,95 \%$ CI 0.5 to $1.01, \mathrm{P}=0.058$ ). The effect on adherence was absent in the 
per protocol analysis that excluded a large number of missing patients (up to $45 \%$ in the control arm and $32 \%$ in the intervention arm). Epidemiologists have cautioned that, in the presence of high or differential losses to follow-up, the intention-to-treat analysis may overestimate the treatment effect. ${ }^{29}$ A second trial from Kenya ${ }^{5}$ tested four types of mobile phone reminders that were either short or long and were sent to patients either daily or weekly. The proportion of adherent patients was reported to be significantly higher in only one of the four intervention groups, with a significance level of 0.03 . If the analysis had incorporated appropriate correction for multiple hypotheses, it is likely that the observed significance may be diminished. Other studies and analyses from Africa have also suggested that additional counseling approaches using telephones can have positive outcomes on ART adherence. ${ }^{30} 31$ These trials nevertheless contribute important evidence that the nature and complexity of the mobile phone communication may be essential to efficacy ${ }^{32}$ Noteworthy advantages of our trial compared with these earlier trials included the longer duration of our trial and the use of more robust measures such as three-monthly viral load assays and standardised, objective measurement of pill count to assess adherence. In addition, missing data and losses to follow-up were minimal in our study, and analysis in the intention-to-treat, per protocol, and subgroup populations were consistent in their lack of significant effect. Although not statistically significant, our results indicate a trend toward lower attrition in the mobile phone group. Overall, our results lend credence to the consideration that mobile phone communications used as reminders for medications alone may not improve adherence and treatment success among HIV infected patients, and suggest instead that the nature of the communication is an important factor for success.

Several other studies in areas without a high prevalence of HIV infection have demonstrated apparent non-effect of text reminders in medication adherence. ${ }^{2}$ Adherence to malaria chemoprophylaxis among French soldiers from Côte d'Ivoire did not increase with daily text message reminders on their mobile phones. ${ }^{33}$ A similar intervention showed no benefit in improving oral contraceptive adherence among women in the US. ${ }^{34} \mathrm{~A}$ well researched systematic review of interventions used for enhancing adherence to medications indicated that unifaceted interventions such as simple reminders had little impact on adherence in chronic health conditions, while successful interventions were complex and multifaceted, often including manual telephone follow-up. ${ }^{32}$

Among the 139 (18\%) patients who declined consent, demographic and clinical features (including age, sex, demographics, education, residence, recruitment site, and primary drug resistance) were similar to those of the patients who participated in the trial. These results show that external validity of the results is likely to be in the acceptable range for these known factors. However it was not possible to compare behavioural characteristics, and hence there remains the possibility that there was a selection bias of non-measurable behavioural characteristics among the trial participants.

\section{Interpretation of study results}

Foremost among possible reasons for the observed lack of effect in our trial is the apparent simplicity of the intervention, as it was designed to function more as a reminder for medications than as a means of open communication between the patient and the healthcare provider. It has become increasingly clear that determinants of adherence $t$ o HIV medication are exceedingly complex, and effective interventions to improve adherence can be developed only to the extent that we comprehend mechanisms underlying behaviour. ${ }^{35}$ Forgetfulness and being away from medications are oft quoted reasons for poor adherence to treatment in many geographical areas. ${ }^{36}$ These response patterns were remarkably consistent within our own setting. ${ }^{37}$

Building on this information, we designed an intervention to remind patients to take their medication and thus sustain treatment success via enhanced adherence. However, "forgetfulness" often masks a lack of engagement in services that a patient adopts either voluntarily or is forced to adopt through objectification and disempowerment; this also indicates that the treatment is minimally meaningful to the patient's subjective health goals. The underlying reasons for those patients who have reported forgetfulness in our setting are likely to be complex and require further exploration. The provision of mobile phone reminders serves as external cues to support adherence. Such an intervention using reminders is based on behavioural learning theory, which focuses on the use of antecedents and their influence on behaviour. ${ }^{38}$ These antecedents could be internal (such as patient perceptions) or external circumstances, as in the case of phone reminders used in our trial.

A criticism of behavioural learning theory has been the lack of an individualised approach and inadequate consideration of other less conscious influences on adherence, such as lack of acceptance of a diagnosis. ${ }^{39}$ Interventions derived from a substantive theory of behaviour change tend to be more effective than are those based on intuition..$^{40}$ Researchers have argued that the rationalist and hierarchical approach cannot hope to address the social processes that underlie decision making with relation to ART. ${ }^{41}$ A deeper consideration of the theory of planned behaviour among South African patients showed that the combination of attitudes towards adherence, perceived behavioural control, and subjective norms explained merely $12 \%$ of ART adherence intentions. ${ }^{42}$ Additionally, the pathway between intention and adherence behaviour is mediated via self regulatory processes. ${ }^{43}$

A model drawn from self regulation theory suggests that patients seek to understand their illness by developing a working model or representation of what the illness is, its cause, its effects, and how the illness can be controlled. Patients may demonstrate better adherence if they receive adequate information that enhances accurate treatment representation, reduces discrepancy between expectations and actual experiences, and facilitates the perception that experience is manageable. ${ }^{43}$ Thus interventions that harness these aspects by including early intense counselling and patients' participatory activities with caring medical staff may have more positive effects on adherence than simple reminders. ${ }^{44}$ Exposure of the control arm to good standard care can be a limiting factor in evaluating the intervention effect. ${ }^{45}$ Thus it is also reasonable to suggest that the existing national system of counselling and clinical support may have contributed substantially towards maintaining a higher level of adherence and self efficacy in our setting. ${ }^{46}$

Next, the appropriate definition of the outcome measure - optimal adherence - is much debated. There is accumulating evidence that with currently available, potent ART, adherence levels of $80 \%$ or lower can be sufficient. ${ }^{47}$ Indeed, recent estimates of optimal adherence for patients taking non-nucleoside reverse transcriptase inhibitor and boosted protease inhibitor therapy have been as low as $54-74 \% .^{48}{ }^{49} \mathrm{Is}$ it time then, to revisit the desired adherence threshold for virological suppression? Using longitudinal cohort data to model the effect of adherence on viral load over different durations of viral suppression, the authors notably concluded that, among 
patients with at least 12 months of viral suppression, a minimum adherence level of $50 \%$ could reduce the probability of virological failure to $2-6 \% .^{50}$ These emerging data may offer partial explanation for the seeming lack of effect of interventions to enhance adherence such as mobile phone reminders within a setting of sufficiently high adherence levels.

\section{Conclusions}

In conclusion, evidence generated by our rigorously designed randomised trial indicates that simple reminders on mobile phones alone may not solve the problem of treatment failure in HIV. These results may be interpreted with caution as the intervention was targeted at reminding patients about taking medication and did not include any additional information or support. Our results do not detract from the tremendous potential of mobile phone technology in healthcare. Rather, mobile phones in conjunction with other strategies, may facilitate communication between healthcare providers and patients that can help improve health outcomes in chronic conditions. ${ }^{51}$ Whether phone reminders specifically translate into improved adherence and treatment success is a question that is still being examined.

All authors are part of the HIVIND (HIV-India) Study Team. Other members of the HIVIND Study Team are: George D'souza, Ujjwal Neogi, Karthika Arumugam, Shubha Krishnamurthy, Jimmy Antony, Soham Gupta, Vijesh Kuttiatt (St John's Medical College Hospital, Bangalore); Selvamuthu Poongulali, Esther Faith Beulah, Kavitha Balaji, Syed lqbal, Shanmugam Saravanan, Chandrasekaran Ezhilarasi (YRGCARE Medical Centre, Chennai); Vasudev Naik, M Srinivasa (Krishna Rajendra Hospital, Mysore); Suresh Shastri, Sunil Dodderi (Karnataka State AIDS Prevention Society); Clas Källander (Cavidi, Sweden, HIVIND Consortium)

We thank the HIVIND Study Team for their outstanding efforts in recruiting, interviewing, and following the study participants. We also acknowledge the staff at the ART Centre at St John's Medical College Hospital, Bangalore, and KR Hospital, Mysore, and the clinical team at YRGCARE, Chennai, for their excellent teamwork and patient care. We thank the National AIDS Control Organisation (NACO), Government of India, for supporting this project. Most importantly, we are grateful to the study subjects for their patience and participation in this trial.

Contributors: AS designed the data collection tools, supervised data collection at the Karnataka site, analysed the data and drafted the paper. $A D C$ coordinated the collaborative trial, led the design of the trial protocol, trained staff and gave input on data collection tool design, monitored data collection, provided critical input for the analysis plan and manuscript draft, and is guarantor for the trial. NK provided input on the study plan, supervised data collection and implemented the trial in Chennai, and provided input to the manuscript. RR supported the development of data collection instruments, worked with training of staff, monitored data collection, supported data analysis, and revised the draft paper. BBR provided input on the study plan and provided critical comments on the paper. PA supported development of the trial protocol, provided critical inputs on the statistical analysis plan, provided quality assurance for the whole trial, and contributed to the manuscript draft. $\mathrm{BE}$ wrote the statistical analysis plan, monitored data collection for the whole trial, and contributed to the manuscript. VD initiated the HIVIND project, steered the HIVIND, provided guidance on the study design analysis and drafting the paper, and is guarantor for the trial. All authors have approved the final submission.

Funding: This study was funded by the European Union, Framework Program 7 (project No 222946). The study sponsor had no role in the study design; collection, analysis, and interpretation of data; writing the report; or in the decision to submit the article for publication.
Competing interests: All authors have completed the ICMJE uniform disclosure form at www.icmje.org/coi_disclosure.pdf and declare: no support from any organisation for the submitted work; no financial relationships with any organisations that might have an interest in the submitted work in the previous three years; no other relationships or activities that could appear to have influenced the submitted work. Ethical approval: Ethical approval for the trial was obtained from all participating institutions before study initiation. In accordance with national requirements and the principles of the Declaration of Helsinki, written informed consent was obtained from all participants before enrolment.

Data sharing: No additional data available.

Transparency: The authors had full access to all of the data (including statistical reports and tables) in the study and take responsibility for the integrity of the data and the accuracy of the data analysis. The guarantor, on behalf of all the authors, affirms that the manuscript is an honest, accurate, and transparent account of the study being reported, and that no important aspects of the study have been omitted, and that all the changes of the study plan have been explained fully and justified.

Catalani C, Philbrick W, Fraser H, Mechael P, Israelski DM. mHealth for HIV treatment and prevention: a systematic review of the literature. Open AIDS J 2013;7:17-41.

2 Free C, Phillips G, Galli L, Watson L, Felix L, Edwards P, et al. The effectiveness of mobile-health technology-based health behaviour change or disease management interventions for health care consumers: a systematic review. PLoS Med 2013;10:e1001362.

3 Free C, Phillips G, Watson L, Galli L, Felix L, Edwards P, et al. The effectiveness of mobile-health technologies to improve health care service delivery processes: a systematic review and meta-analysis. PLoS Med 2013; 10(1): e1001363.

4 Lester RT, Ritvo P, Mills EJ, Kariri A, Karanja S, Chung MH, et al. Effects of a mobile phone short message service on antiretroviral treatment adherence in Kenya (WeITel Kenya1): a randomised trial. Lancet 2010; 376(9755): 1838-45.

5 Pop-Eleches C, Thirumurthy H, Habyarimana JP, Zivin JG, Goldstein MP, de Walque D, et al. Mobile phone technologies improve adherence to antiretroviral treatment in a resource-limited setting: a randomized controlled trial of text message reminders. AIDS 2011; 25(6): 825-34.

6 Horvath T, Azman H, Kennedy GE, Rutherford GW. Mobile phone text messaging for promoting adherence to antiretroviral therapy in patients with HIV infection. The Cochrane database of systematic reviews 2012; 3: CD009756.

7 World Health Organization. Consolidated guidelines on the use of antiretroviral drugs for treating and preventing HIV infection: recommendations for a public health approach. June 2013. Available at http://www.who.int/hiv/pub/guidelines/arv2013/download/en/index. html. Geneva: WHO 2013.

8 Mbuagbaw L, Thabane L, Ongolo-Zogo P, Lester RT, Mills EJ, Smieja M, et al. The Cameroon Mobile Phone SMS (CAMPS) trial: a randomized trial of text messaging versus usual care for adherence to antiretroviral therapy. PLoS One 2012; 7(12): e46909.

9 Da Costa TM, Barbosa BJ, Gomes e Costa DA, Sigulem D, de Fatima Marin H, Filho AC et al. Results of a randomized controlled trial to assess the effects of a mobile SMS-based intervention on treatment adherence in HIV/AIDS-infected Brazilian women and impressions and satisfaction with respect to incoming messages. Int J Med Inform 2012; 81(4): 257-69.

10 Tomlinson M, Rotheram-Borus MJ, Swartz L, Tsai AC. Scaling up mHealth: where is the evidence? PLoS Med 2013; 10(2): e1001382.

11 National AIDS Control Organisation. Annual Report, 2012-2013. Department of AIDS Control. Ministry of Health and Family Welfare, New Delhi. Available at http://www.naco. gov.in/NACO/Quick Links/Publication/Annual Report/NACO Annual Report/Annual Report_2012-13/. 2013.

12 Ajzen I. The theory of planned behaviour: reactions and reflections. Psychol Health 2011; 26(9): 1113-27.

13 Shet A, Arumugam K, Rodrigues R, Rajagopalan N, Shubha K, Raj T, et al. Designing a mobile phone-based intervention to promote adherence to antiretroviral therapy in South India. AIDS Behav 2010; 14(3): 716-20.

14 Rodrigues R, Shet A, Antony J, Sidney K, Arumugam K, Krishnamurthy S, et al. Supporting adherence to antiretroviral therapy with mobile phone reminders: results from a cohort in South India. PLoS One 2012; 7(8): e40723.

15 De Costa A, Shet A, Kumarasamy N, Ashorn P, Eriksson B, Bogg L, et al. Design of a randomized trial to evaluate the influence of mobile phone reminders on adherence to first line antiretroviral treatment in South India--the HIVIND study protocol. BMC Med Res Methodol 2010; 10: 25.

16 HIVIND: The antiretroviral roll-out for HIV in India, Available at http://hivind.eu/, Accessed on 6 December 2013. 2013

17 National AIDS Control Organization. Antiretroviral therapy guidelines for HIV-infected adults and adolescents including post-exposure prophylaxis. Available at http://www.naco. gov.in/NACO/About_NACO/Policy_Guidelines/Policies_Guidelines1/New Delhi: Ministry of Health \& Family Welfare, Government of India; 2007.

18 Lampe FC, Gatell JM, Staszewski S, Johnson MA, Pradier C, Gill MJ, et al. Changes over time in risk of initial virological failure of combination antiretroviral therapy: a multicohort analysis, 1996 to 2002. Arch Intern Med 2006; 166(5): 521-8.

19 Bachani D, Garg R, Rewari BB, Hegg L, Rajasekaran S, Deshpande A, et al. Two-year treatment outcomes of patients enrolled in India's national first-line antiretroviral therapy programme. Nat/ Med J India 2010; 23(1): 7-12.

20 Alvarez-Uria G, Naik PK, Pakam R, Midde M. Factors associated with attrition, mortality, and loss to follow up after antiretroviral therapy initiation: data from an HIV cohort study in India. Global health action 2013; 6: 21682. 


\section{What is already known on this subject}

The use of mobile phones in healthcare in increasing; in HIV care, mobile phones have been used with mixed success to improve retention in care and adherence to medication

Whether this translates to improved virological suppression and treatment success is not clear: there is limited evidence from sub-Saharan Africa, but no evidence from Asia

\section{What this study adds}

This randomised controlled trial suggests that mobile phone reminders have no overall benefit in improving adherence to antiretroviral therapy or virological suppression among Indian patients starting therapy

21 Barth RE, van der Loeff MF, Schuurman R, Hoepelman AI, Wensing AM. Virological follow-up of adult patients in antiretroviral treatment programmes in sub-Saharan Africa: a systematic review. Lancet Infect Dis 2010; 10(3): 155-66.

22 De Beaudrap P, Thiam M, Diouf A, Toure-Kane C, Ngom-Gueye NF, Vidal N, et al. Risk of virological failure and drug resistance during first and second-line antiretroviral therapy in a 10-year cohort in Senegal: results from the ANRS 1215 cohort. J Acquir Immune Defic Syndr 2013; 62(4): 381-7.

23 Aghokeng AF, Monleau M, Eymard-Duvernay S, Dagnra A, Kania D, Ngo-Giang-Huong $\mathrm{N}$, et al. Extraordinary heterogeneity of virological outcomes in patients receiving highly $\mathrm{N}$, et al. Extraordinary heterogeneity of virological outcomes in patients receiving highly aptroach in sub-saharan Africa and southeast Asia. Clin Infect Dis 2014; 58(1): 99-109.

24 San Lio MM, Carbini R, Germano P, Guidotti G, Mancinelli S, Magid NA, et al. Evaluating adherence to highly active antiretroviral therapy with use of pill counts and viral load measurement in the drug resources enhancement against AIDS and malnutrition program in Mozambique. Clin Infect Dis 2008; 46(10): 1609-16.

25 Gokarn A, Narkhede MG, Pardeshi GS, Doibale MK. Adherence to antiretroviral therapy $J$ Assoc Physicians India 2012; 60: 16-20.

26 Mhaskar R, Alandikar V, Emmanuel P, Djulbegovic B, Patel S, Patel A, et al. Adherence to antiretroviral therapy in India: a systematic review and meta-analysis. Indian $J$ Community Med 2013; 38(2): 74-82.

27 McCambridge J, Witton J, Elbourne DR. Systematic review of the Hawthorne effect: New concepts are needed to study research participation effects. J Clin Epidemiol 2013.

28 McCambridge J, Kypri K, Elbourne D. In randomization we trust? There are overlooked problems in experimenting with people in behavioral intervention trials. J Clin Epidemiol 2014; 67(3): 247-53.

29 Hernan MA, Hernandez-Diaz S. Beyond the intention-to-treat in comparative effectiveness research. Clin Trials 2012; 9(1): 48-55.

30 Mbuagbaw L, van der Kop ML, Lester RT, Thirumurthy $\mathrm{H}$, Pop-Eleches C, Ye C, et al. Mobile phone text messages for improving adherence to antiretroviral therapy (ART): an individual patient data meta-analysis of randomised trials. BMJ open 2013; 3(12): e003950.

31 Maduka O, Tobin-West Cl. Adherence counseling and reminder text messages improve uptake of antiretroviral therapy in a tertiary hospital in Nigeria. Niger J Clin Pract 2013; 16(3): 302-8.

32 Haynes RB, Ackloo E, Sahota N, McDonald HP, Yao X. Interventions for enhancing medication adherence. The Cochrane database of systematic reviews 2008; (2): CD000011.

33 Ollivier L, Romand O, Marimoutou C, Michel R, Pognant C, Todesco A, et al. Use of short message service (SMS) to improve malaria chemoprophylaxis compliance after returning from a malaria endemic area. Malar J 2009; 8: 236

34 Hou MY, Hurwitz S, Kavanagh E, Fortin J, Goldberg AB. Using daily text-message reminders to improve adherence with oral contraceptives: a randomized controlled trial. Obstet Gynecol 2010; 116(3): 633-40.

35 Amico KR, Harman JJ, Johnson BT. Efficacy of antiretroviral therapy adherence interventions: a research synthesis of trials, 1996 to 2004. J Acquir Immune Defic Synd 2006; 41(3): 285-97.

36 Mills EJ, Nachega JB, Bangsberg DR, Singh S, Rachlis B, Wu P, et al. Adherence to HAART: a systematic review of developed and developing nation patient-reported barriers and facilitators. PLoS Med 2006; 3(11): e438.
37 Ekstrand ML, Shet A, Chandy S, Singh G, Shamsundar R, Madhavan V, et al. Suboptimal adherence associated with virological failure and resistance mutations to first-line highly active antiretroviral therapy (HAART) in Bangalore, India. Int Health 2011; 3(1): 27-34.

38 Munro S, Lewin S, Swart T, Volmink J A review of health behaviour theories: how useful are these for developing interventions to promote long-term medication adherence for TB and HIV/AIDS? BMC Public Health 2007; 7: 104

39 Blackwell B. Compliance. Psychother Psychosom 1992; 58(3-4): 161-9.

40 Fisher JD, Cornman DH, Norton WE, Fisher WA. Involving behavioral scientists, health care providers, and HIV-infected patients as collaborators in theory-based HIV prevention and and antiretroviral adherence interventions. J Acquir Immune Defic Syndr 2006; 43 Supp 1. S10-7.

41 Mykhalovskiy E. Beyond decision making: class, community organizations, and the healthwork of people living with HIV/AIDS. Contributions from institutional ethnographic research. Med Anthropol 2008; 27(2): 136-63.

42 Saal W, Kagee A. The applicability of the Theory of Planned Behaviour in predicting adherence to ART among a South African sample. J Health Psychol 2012; 17(3): 362-70.

43 Reynolds NR. The problem of antiretroviral adherence: a self-regulatory model for intervention. AIDS Care 2003; 15(1): 117-24.

44 Rueda S, Park-Wyllie LY, Bayoumi AM, Tynan AM, Antoniou TA, Rourke SB, et al. Patient support and education for promoting adherence to highly active antiretroviral therapy for HIV/AIDS. The Cochrane database of systematic reviews 2006; (3): CD001442.

45 de Bruin M, Viechtbauer W, Schaalma HP, Kok G, Abraham C, Hospers HJ. Standard care impact on effects of highly active antiretroviral therapy adherence interventions: A meta-analysis of randomized controlled trials. Arch Intern Med 2010; 170(3): 240-50.

46 Shet A, DeCosta A, Heylen E, Shastri S, Chandy S, Ekstrand M. High rates of adherence and treatment success in a public and public-private HIV clinic in India: potential benefits of standardized national care delivery systems. BMC Health Serv Res 2011; 11: 277.

47 Kobin AB, Sheth NU. Levels of adherence required for virologic suppression among newe antiretroviral medications. Ann Pharmacother 2011; 45(3): 372-9.

48 Bangsberg DR. Less than $95 \%$ adherence to nonnucleoside reverse-transcriptase inhibitor therapy can lead to viral suppression. Clin Infect Dis 2006; 43(7): 939-41.

49 Shuter J, Sarlo JA, Kanmaz TJ, Rode RA, Zingman BS. HIV-infected patients receiving lopinavir/ritonavir-based antiretroviral therapy achieve high rates of virologic suppression despite adhence

50 Rosenblum M, Deeks SG, van der Laan M, Bangsberg DR. The risk of virologic failure decreases with duration of HIV suppression, at greater than $50 \%$ adherence to antiretroviral therapy. PLoS One 2009; 4(9): e7196.

51 Lester RT. Ask, don't tell - mobile phones to improve HIV care. N Engl J Med 2013 369(19): 1867-8.

\section{Cite this as: BMJ 2014;349:95978}

This is an Open Access article distributed in accordance with the Creative Commons Attribution Non Commercial (CC BY-NC 4.0) license, which permits others to distribute, remix, adapt, build upon this work non-commercially, and license their derivative works on different terms, provided the original work is properly cited and the use is non-commercial. See: http://creativecommons.org/licenses/by-nc/4.0/. 


\section{Tables}

Table 1| Baseline social, demographic, and clinical characteristics of HIV infected patients before randomisation to intervention or control. Values are numbers (percentages) of patients unless specified otherwise

\begin{tabular}{|c|c|c|}
\hline Characteristic & Mobile phone intervention arm ( $n=315)$ & Standard care control arm $(n=316)$ \\
\hline \multicolumn{3}{|l|}{ Social and demographic characteristics } \\
\hline Female & $136(43.2)$ & $137(43.4)$ \\
\hline \multicolumn{3}{|l|}{ Age (years): } \\
\hline $18-30 \mathrm{yrs}$ & $76(24.1)$ & $79(25.0)$ \\
\hline $31-40$ yrs & $150(47.6)$ & $156(49.4)$ \\
\hline $41-60$ yrs & $89(28.3)$ & $81(25.6)$ \\
\hline \multicolumn{3}{|l|}{ Education: } \\
\hline No formal education & $59(18.7)$ & $57(18.0)$ \\
\hline Up to high school & $189(60.0)$ & $190(60.1)$ \\
\hline Beyond high school & $67(21.3)$ & $69(21.8)$ \\
\hline Literate $^{*}$ & $252(80.0)$ & $250(79.1)$ \\
\hline Rural residence ( $v$ urban) & $143(45.4)$ & $143(45.3)$ \\
\hline Currently married & $217(68.9)$ & $218(69.0)$ \\
\hline Unemployed or household duties ( $v$ employed) & $116(36.8)$ & $117(37.0)$ \\
\hline Access to mobile phone & $263(83.5)$ & $260(82.3)$ \\
\hline Previous ownership of mobile phone & $246(78.1)$ & $249(78.8)$ \\
\hline Household income $\leq \$ 1000$ per year & $229(72.7)$ & $237(75.0)$ \\
\hline \multicolumn{3}{|l|}{ Recruiting sites: } \\
\hline Bangalore & $81(25.7)$ & $77(24.4)$ \\
\hline Mysore & $81(25.7)$ & $83(26.3)$ \\
\hline Chennai & $153(48.6)$ & $156(49.4)$ \\
\hline \multicolumn{3}{|l|}{ Clinical characteristics } \\
\hline WHO* clinical staging 3 and $4 \dagger$ & $175(55.6)$ & $170(53.8)$ \\
\hline CD4 cell count $<250 \times 10^{6} / \mathrm{L}$ & $230(73.0)$ & $217(68.7)$ \\
\hline Median (IQR) CD4 cell count (cells $\left.\times 10^{6} / \mathrm{L}\right)$ & $185(97-253)$ & $193(115-268)$ \\
\hline Median (IQR) baseline viral load ( $\log _{10}$ copies $/ \mathrm{mL}$ ) & $5.5(5.1-6.0)$ & $5.4(4.9-5.9)$ \\
\hline ART regimen: & $(n=305) \ddagger$ & $(n=308) \ddagger$ \\
\hline Zidovudine based & $136(44.6)$ & $133(43.2)$ \\
\hline Stavudine based & 34 (11.2) & $38(12.3)$ \\
\hline Tenofovir based & $135(44.1)$ & $137(44.5)$ \\
\hline Baseline transmitted drug resistance & $13 / 309(4.2)$ & $12 / 308(3.9)$ \\
\hline
\end{tabular}

IQR=interquartile range, ART=antiretroviral treatment.

${ }^{*}$ Can read or write in local language (Kannada, Tamil, Telugu, Hindi).

†World Health Organization clinical staging of HIV/AIDS: stages 3 and 4 indicate advanced HIV infection defined clinically.

$\ddagger 10$ patients in intervention arm and 8 in control arm died or withdrew from study. 
Table 2| Overview of primary and secondary outcomes in trial of HIV infected patients starting antiretroviral treatment (ART) and assigned to mobile phone intervention or standard care

\begin{tabular}{|c|c|c|c|c|c|c|}
\hline & \multicolumn{2}{|c|}{ No $(\%)$ of patients } & \multicolumn{2}{|c|}{ Unadjusted analysis } & \multicolumn{2}{|c|}{ Adjusted analysis* } \\
\hline & $\begin{array}{l}\text { Mobile phone } \\
\text { intervention }\end{array}$ & $\begin{array}{c}\text { Standard care } \\
\text { control }\end{array}$ & Ratio $(95 \% \mathrm{Cl}) \dagger$ & $P$ value & Ratio (95\% Cl)† & $P$ value \\
\hline Primary outcome & & & \multicolumn{2}{|c|}{ Hazard ratio } & \multicolumn{2}{|c|}{ Hazard ratio } \\
\hline Virological failure & 49/315 (15.6) & 49/316 (15.5) & 0.98 (0.67 to 1.47$)$ & 0.95 & 0.96 (0.65 to 1.43$)$ & 0.85 \\
\hline $\begin{array}{l}\text { Secondary } \\
\text { outcomes }\end{array}$ & & & \multicolumn{2}{|c|}{ Incidence rate ratio } & \multicolumn{2}{|c|}{ Incidence rate ratio } \\
\hline $\begin{array}{l}\text { Suboptimal } \\
\text { adherence to } \\
\text { ART‡ }\end{array}$ & $81 / 300(27.0)$ & 65/299 (21.7) & 1.24 (0.93 to 1.65$)$ & 0.14 & 1.24 (0.94 to 1.63$)$ & 0.13 \\
\hline Death & $21 / 315(6.7)$ & 23/316 (7.3) & 0.92 (0.52 to 1.62$)$ & 0.76 & 0.91 (0.51 to 1.60$)$ & 0.74 \\
\hline Missing & $24 / 315(7.6)$ & $30 / 316$ (9.5) & $0.80(0.48$ to 1.34$)$ & 0.40 & $0.59(0.32$ to 1.10$)$ & 0.10 \\
\hline
\end{tabular}

*Adjustment for recruitment site; other sociodemographic confounders such as age, sex, rural residence, education level, previous experience with mobile phones; baseline CD4 count; ART regimen; occurrence of adverse drug reactions; and presence of transmitted drug resistance at baseline.

†Hazard ratio used for primary outcome, incidence rate ratio used for secondary outcomes.

$\ddagger$ Mean adherence $<95 \%$ by pill count. 


\section{Figures}

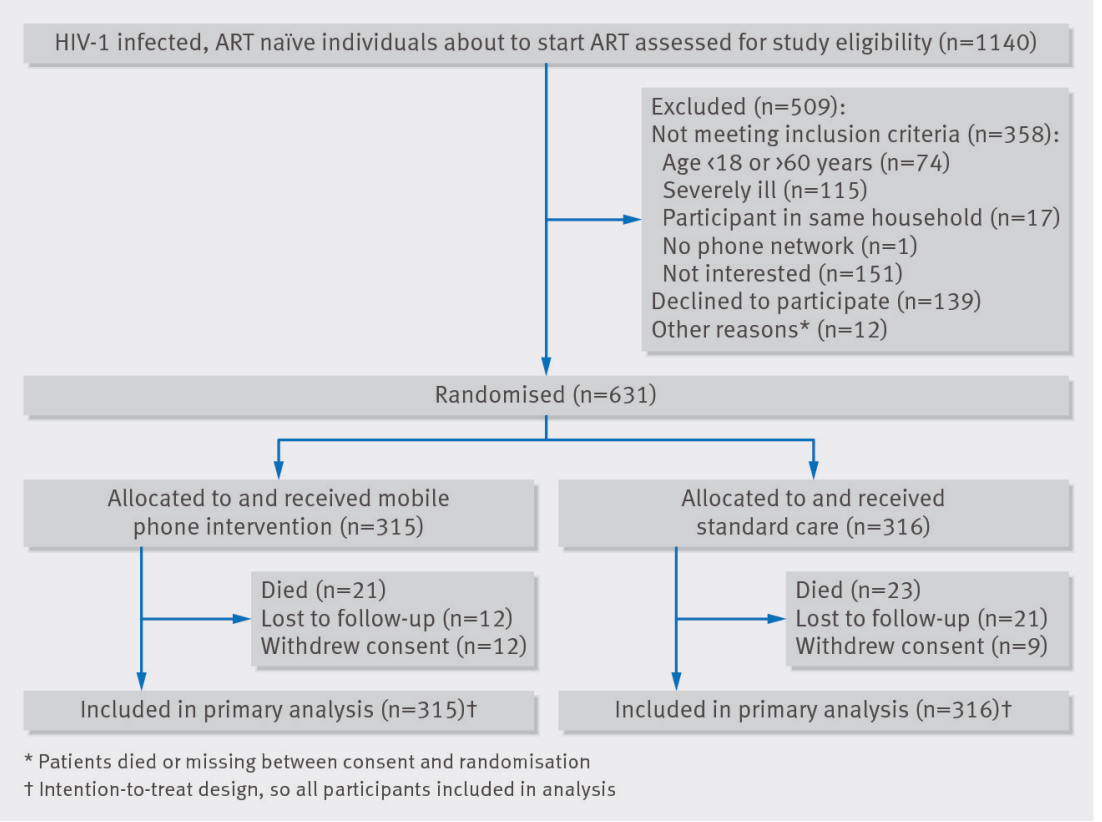

Fig 1 Flow diagram of study design and patient enrolment

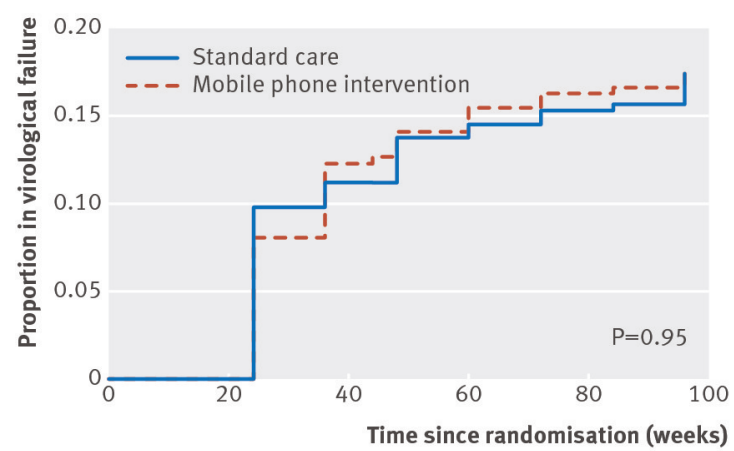

No at risk (No of virological failures)

Standard care

$$
308(0) \quad 287(32) \quad 242(7) \quad 231(4) \quad 222(6) \quad 0
$$

Mobile phone intervention

$307(0) \quad 289(35) \quad 245(5) \quad 238(6) \quad 228(3) \quad 0$

Fig 2 Proportion of patients experiencing virological failure in the intervention and standard care arms over time (Kaplan-Meier estimates of 2 year cumulative hazard rates of virological failure among patients initiating antiretroviral therapy and assigned to mobile phone intervention or standard care). Participants who died, withdrew consent, or went missing were right censored. The overall adjusted hazard ratio for virological failure was $0.96(95 \% \mathrm{Cl} 0.65$ to 3.43$)$. Also shown are the number of patients at risk and the number of virological failures at each time point. The $P$ value was calculated by means of Cox regression. 


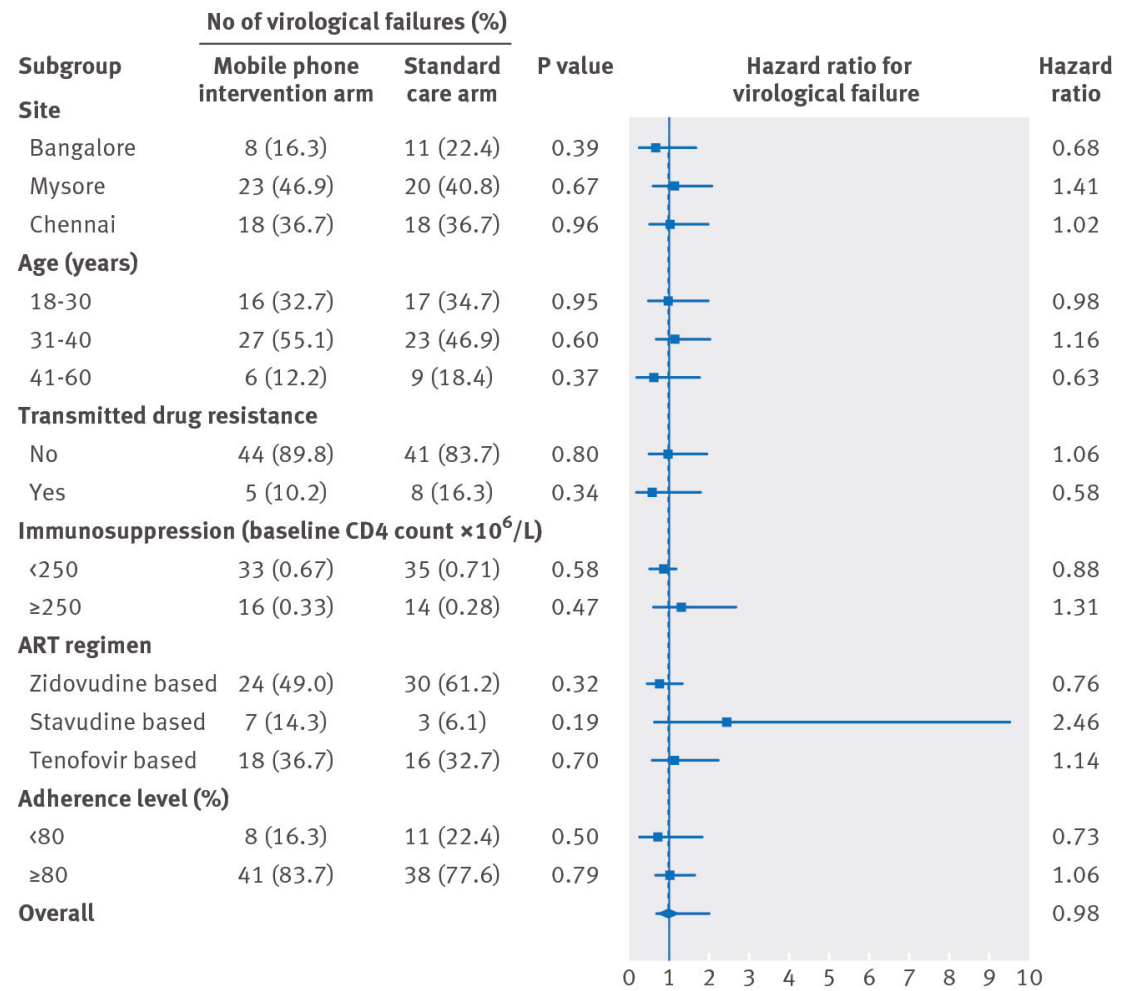

Fig 3 Subgroup analysis of outcomes. Hazard ratios for virological failure using Cox regression analysis are shown for each subgroups 